\title{
INSTITUTO COMPLIANCE NO BRASIL E A EFICÁCIA NA MITIGAÇÃO AO RISCO COR- PORATIVO $^{1}$
}

\section{INSTITUTE COMPLIANCE IN BRAZIL AND EFFICIENCY IN MITIGATION TO CORPO- RATE RISK}

\author{
Hildegardo Pedro Araujo de Melo \\ Mestre em Controladoria pela UFRPE \\ Ministério Público de Pernambuco \\ hildegardomelo70@gmail.com
}

\author{
Adilson Celestino de Lima \\ doutorado em Administração pela UFPE \\ Universidade Federal Rural de Pernambuco \\ celestinolima@yahoo.com.br
}

\section{RESUMO}

Objetivo: Investigar a relação entre as práticas de compliance e sua eficácia na resposta aos riscos corporativos no Brasil.

Fundamento: Neste trabalho, a discussão partiu da análise do comportamento dos profissionais de organizações brasileiras, quanto à capacidade organizacional de resposta a fraudes e riscos regulatórios sob o prisma da orientação COSO ERM (2007), considerando a probabilidade e o impacto da ocorrência do evento.

Método: Baseado no pressuposto de que compliance representa um fator preponderante na redução aos riscos, foram aplicados testes para medir a capacidade de resposta a eventos de fraudes e regulatórios diante de uma cultura de compliance disseminada, através de uma amostra composta por dois grupos distintos de profissionais, como forma de ampliar o potencial explicativo do estudo e possibilitar a possível influência da característica desta amostra na capacidade de resposta aos riscos.

Resultados: Foram encontradas diferenças significativas para estrutura de compliance entre os grupos da amostra, porém não existiram diferenças estatísticas em relação à capacidade de resposta ao risco. Nos resultados da distribuição dos dados por grupo amostral, o comportamento da amostra está aquém do esperado, quando relacionados à característica da população a frequência da suscetibilidade de ocorrência dos riscos.

${ }^{1}$ Artigo recebido em: 27/08/2018. Revisado por pares em: 27/11/2018. Reformulado em: 31/07/2019. Recomendado para publicação em: 09/08/2019 por Adriana Fernandes de Vasconcelos (Editora Geral). Publicado em: 02/09/2019. Organização responsável pelo periódico: UFPB 
Contribuições: $\mathrm{O}$ estudo contribui para ampliar a discussão sobre a aplicabilidade e os ganhos do efetivo compliance, como instrumento de governança para a gestão de risco na formação de um ambiente de conformidade com a defesa de valores intrínsecos para a transformação da realidade organizacional.

Palavras-Chave: Compliance no Brasil. Eficácia. Resposta. Riscos Corporativos.

\begin{abstract}
Purpose: Investigate the relationship between compliance practices and their effectiveness in responding to corporate risks in Brazil.
\end{abstract}

Foundation: In this paper, the discussion came from the analysis of the behavior of professionals from Brazilian organizations, regarding the organizational capacity to respond to fraud and regulatory risks from the perspective of COSO ERM (2007), considering the probability and impact of the event.

Method: Based on the assumption that compliance is a major factor in risk reduction, tests were performed to measure the response to fraud and regulatory events in the face of a widespread compliance culture, through a sample of two distinct groups professionals, as a way to expand the explanatory potential of the study and enable the possible influence of the characteristics of this sample on the risk response capacity.

Results: Were found significant differences for compliance structure between the sample groups, but there were no statistical differences regarding the risk response capacity. In the results of the distribution of data by sample group, the behavior of the sample is lower than expected, when related to the characteristic of the population the frequency of susceptibility of risks occurrence.

Contributions: The study contributes to broaden the discussion on the applicability and gains of effective compliance, as a governance instrument for risk management in the formation of an environment of compliance with the defense of intrinsic values for the transformation of organizational reality.

Keywords: Compliance in Brazil. Efficiency. Answer. Corporate Risks.

\title{
1 INTRODUÇÃO
}

Compliance está relacionado ao comprometimento organizacional, atendimento aos interesses preestabelecidos e à ética organizacional que transforma a realidade do ambiente. Nesse ponto, compliance é compreendido, antes de tudo, como um estado de agir (Tarantino, 2008).

Essa compreensão justifica a ideia capturada do meio corporativo de que o compliance pode representar uma estratégia à disposição da organização para a mitigação dos riscos e prevenção da fraude (Costa, 2012). Este raciocínio corrobora com os achados de Laruccia e Yamada (2011) que apresentam compliance inserido na organização, como controle na mitigação de riscos através da construção de políticas e normas com repercussão nas práticas saudáveis para a gestão de riscos.

A alusão ao tema sempre esteve atrelado aos fatos históricos da literatura que remontam aos grandes escândalos financeiros do final da década de 90, logo, compliance está associado à medida de fortalecimento do sistema de controle interno organizacional para mitigação da ocorrência de riscos e disseminar a cultura de controles para assegurar o cumprimento de leis e regulamentos (ABBI \& FEBRABAN, 2009).

A exposição aos riscos derivados de falhas nos processos internos e fraudes correspondem pela maior parte das incertezas no mercado (KPMG, 2015a; KPMG, 2015b), logo, os esforços de instrumentos de governança tem se mostrado cada vez mais preocupados para o controle efetivo 
da ocorrência destes riscos, trazendo a partir da revisão e fortalecimento dos sistemas de controle o atingimento esperado da conformidade das regras associadas à implementação de práticas que superem problemas que envolvem tais eventos.

Todavia, a partir dos regulamentos internacionais, como Foreing Corrupt Practices Act [FCPA] e a Anti Bribery Act, a prevenção e o combate às fraudes corporativas foram tratados de maneira institucional, exigindo programas selados em sistemas de controle para a regulação e promoção da integridade empresarial.

No Brasil, a Lei 12.846/2013, denominada Lei Anticorrupção, adota as mesmas premissas das referências internacionais de para o enfrentamento dosa crimes de corrupção e fraudes corporativas, aplicáveis às corporações brasileiras por meio da implementação de programas de integridade.

A partir deste marco regulatório, os efeitos da alavancagem de compliance no Brasil ganham maior visibilidade social e acadêmica no País ao fomentar ações de combate a corrupção e fraudes, através de programas formais de integridade preestabelecidos (Trapp, 2015). As organizações brasileiras passaram a demandar maior atenção para implantação de instrumentos que atribuam melhor segurança no alcance de seus objetivos, como o próprio programa de compliance.

Em meio a esta conjuntura é que se encontram mais difundidos os estudos sobre compliance. Contudo, a pouca abordagem de estudos acadêmicos que investiguem se, de fato, a aplicabilidade de compliance no País tem se tornado uma ferramenta eficaz na ação mitigadora do risco e transformadora da cultura de integridade na organização, justificam a realização da pesquisa.

Logo, a questão central que se levanta neste estudo trata saber: Qual a relação entre as práticas de compliance e a capacidade organizacional de resposta aos riscos corporativos? Este estudo tem como objetivo investigar a relação entre as práticas de compliance e sua eficácia na resposta aos riscos corporativos no Brasil.

A pesquisa contribui para ampliar a discussão sobre a aplicabilidade e o ganho do efetivo compliance, na qualidade de instrumento de governança para a gestão de risco e formação de um ambiente de conformidade em defesa dos valores e fortalecimento ético para a transformação da realidade organizacional.

\section{REVISÃO DA LITERATURA}

\subsection{Instituto Compliance}

A prevenção e o combate à corrupção são temas amplamente difundidos por programas institucionais selados em sistemas de controle através de institutos legais para a regulação dos organismos corporativos na promoção da integridade empresarial. Nesse caso, referências como o Foreing Corrupt Practices Act [FCP], aprovado em 1977 pelo Congresso Norte-Americano, uma das principais sustentações normativas de programas de integridade na atualidade e a Anti Bribery Act, lei britânica publicada recentemente (2010) e de grande extensão por sua aplicabilidade nos países do Reino Unido, vem sendo considerada uma das mais severas no combate à corrupção corporativa.

A figura do compliance tem sua origem a partir desses dois conjuntos normativos, compreendidos como grupo de normas positivadas para regulação de relações internas e externas das pessoas jurídicas, bem como daqueles que as compõem, a fim de alcançar a preservação da ética e da moral corporativa (Trapp, 2015).

No cenário nacional, a Lei 12.846/2013 também conhecida como a Lei Anticorrupção surge para resolver uma lacuna no Direito brasileiro ante a ausência legal do instituto próprio para a 
resolução de questões condizentes a responsabilização de pessoas jurídicas por atos impróprios e antiéticos praticados.

Trapp (2015) faz uma relação positiva entre o instituto de compliance e a Lei 12.846/13, apresentando uma complementariedade do segundo pelo primeiro, embora ambos sejam distintos. É que a Lei inova incorporando a figura do compliance ao meio jurídico de forma concreta, impondo a necessidade da inclusão de programas de integridade (compliance) para que as empresas mitiguem os desvios de condutas de seus membros, minimizando os riscos de punições decorrentes de atos ilícitos praticados.

A Lei Anticorrupção prevê a avaliação dos programas de compliance implementados pelas empresas brasileiras a cargo do poder público em regulamento específico. Porém, ainda não foi publicada nenhuma regulamentação sobre o assunto, mesmo a lei tendo entrado em vigor desde 2014.

Uma alternativa para as empresas que operam aqui no Brasil tem sido as recomendações da Controladoria-Geral da União [CGU] às empresas que desejam integrar o rol das organizações que compartilham o combate à corrupção no meio corporativo nacional e comprovem que são íntegras através do Cadastro Pro Ética, ano 2016.

O conjunto de exigências do Cadastro Pro Ética da CGU (aproximadamente 50 itens) atende às práticas gerais necessárias a um programa de compliance, às cobranças trazidas pela Lei Anticorrupção, bem como se ampara aos principais estudos e compilações sobre normas de integridade organizacionais, como o Anti-Corruption Ethics and Compliance Handbook for Business, em 2013 e o Good Practice Guidance on Internal Controls, Ethics and Compliance, 2010, ambas editadas pela Organização para a Cooperação e Desenvolvimento Econômico (OCDE) (Trapp, 2015).

Com isso, as recomendações trazidas pela CGU (2015) na prevenção e combate à corrupção se aproximam às principais referências internacionais sobre o assunto, bem como às exigências da Lei Anticorrupção (Lei no 12.846/2013), do qual guardam entre si pontos centrais, como sugere Ayres (2014, como citado em Xavier, 2015): (i) suporte da alta administração; (ii) análise de risco; (iii) políticas e procedimentos; (iv) comunicação e treinamento; (v) due diligence; (vi) canais de denúncia e investigação internas e (vii) revisão periódica.

Todavia, existem alguns questionamentos na consideração desses parâmetros, especialmente, por demonstrar o comprometimento da organização baseado em incentivos extrínsecos à empresa, como a redução de penalidade pela presença ou não de elementos preestabelecidos da norma, mesmo que tais diretrizes ofereçam elementos essenciais que devem estar contidos em qualquer sistema de prevenção aos atos ilícitos (Figueiredo, 2015).

Assim, importante frisar a discussão feita por Wellner $(2005$, p. 513) para a efetividade de um programa de compliance que deve se basear na definição de valores organizacionais e comprometimento ético, o modelo values-based, em contraponto às premissas como o sugerido pelo modelo norte-americano US Sentencing Guidelines.

Em sua obra, o autor afirmou que pesquisas empíricas sugerem que o values-based Compliance Program é o mais efetivo para deter práticas corruptas, e questiona os benefícios do checklist (box-ticking ou one fits all approach to Compliance) do modelo americano, os quais criam distorções na medida em que (i) encorajam as corporações a adotarem programas subotimos, (ii) privilegiam executivos da alta hierarquia, e (iii) desencorajam pequenas e médias empresas devido ao custo de implementação do programa.

Stucke (2013) concorda com esse pensamento de que o importante para tornar um programa de compliance efetivo são os esforços adotados pela corporação de maneira razoável para asse- 
gurar o cumprimento da lei. Os dois autores defendem, com base em pesquisas empíricas, que o mais eficiente modelo de compliance é aquele que tem como premissa o desenvolvimento de uma cultura organizacional que se baseia em valores éticos.

Uma cultura baseada em valores não diminui a alta performance da corporação, ao invés disso proporciona uma vantagem competitiva para ela (Stucke, 2013, p. 10). Ou seja, conforme cita Xavier (2015), a empresa quer de fato ser ética, e não apenas se proteger das penalidades impostas por leis.

Esses achados corroboram com os estudos anteriores de Katz e Kahn (1974 como citado em Porto \& Tamayo, 2005), de que organizações que se baseiam exclusivamente em formalidade prescrita possuem uma estrutura extremamente frágil. De acordo com os autores, elementos estruturantes do comportamento constituem um desempenho acima dos requisitos de papel para a consecução dos objetivos e funções organizacionais.

\subsection{Disposição para Resposta ao Risco}

Inicialmente, define-se risco, segundo a NBR ISO 31000 (2009), como todo efeito de incerteza nos objetivos da organização, sendo esse efeito um desvio em relação ao planejado, seja ele positivo, seja negativo. A incerteza é o estado, mesmo que parcial, da deformação das informações relacionadas ao evento.

O simples fato de existir atividade corporativa, abre a possibilidade de ocorrência de eventos ou situações cujas consequências constituem oportunidades para obtenção de vantagens (lado positivo) ou ameaças ao sucesso (lado negativo).

Como bem lembra Bergamini Junior (2005), risco é um fato da vida corporativa. Portanto, as empresas devem adotar medidas para geri-lo, quando se tem origem na própria empresa.

Essa definição de risco apresentada por Bergamini Junior (2005), de acordo com Dantas, Rodrigues, Marcellino e Lustosa (2010), é coerente com os preceitos do documento emitido pelo Committee of Sponsoring Organizations of the Treadway Commission (COSO), organização privada criada nos EUA em 1985 para prevenir e evitar fraudes nas demonstrações contábeis das empresas.

A proposta do COSO ERM estabelece medidas para o tratamento adequado ao risco, sendo ampla e aplicável às organizações por meio de conceitos importantes de como manter o risco compatível com o apetite da organização.

Segundo Famá, Cardoso e Mendonça (2003), o gerenciamento de risco se refere à procura por eventos que possam trazer consequências financeiras adversas para a organização, promovendo medidas para salvaguardar a entidade dos riscos causados por tais eventos, prevenindo-os ou minimizando sua ocorrência.

Dessa forma, a existência de estágios preponderantes para a administração do risco nos negócios, que leva em consideração: a) a identificação do risco enfrentado pela empresa; b) a medida do impacto potencial do risco identificado; e c) a decisão se cada risco relevante pode ser minimizado (Famá, Cardoso \& Mendonça, 2003).

Está-se a tratar, de acordo com Brito (2007), a etapa do processo de tratamento aos riscos após a identificação e a avaliação dos eventos considerados inaceitáveis pela organização, segundo a probabilidade de ocorrência dos eventos.

$\mathrm{Na}$ consideração da resposta adequada, a administração avalia o efeito sobre a probabilidade de ocorrência e o impacto do risco, para que a resposta selecionada mantenha os riscos dentro da tolerância desejada. É a orientação do COSO ERM (2007) para o tipo de resposta no tratamento adequado ao risco: evitar, reduzir (mitigar), compartilhar ou aceitar os riscos. 
Logo, é imprescindível a análise sobre a capacidade de resposta aos riscos de fraudes e regulatórios, pois esses eventos causam grande impacto sobre a estrutura organizacional com estragos financeiros, de imagem e quebra do ambiente interno. Por isso, são considerados os riscos mais relevantes à função compliance no Brasil (KPMG, 2015a; KPMG, 2015b).

É a própria resposta à redução da probabilidade de ocorrência do risco por meio do compliance disseminado, para reconhecer que este instituto representa um fator de preponderância no processo de mitigação aos eventos.

É o que se espera de compliance por suas ações de combate e prevenção aos riscos considerado uma solução estratégica disponível, conforme explicam Perera, De Freitas e Imoniana (2014) e Costa (2012). Embora, reconheça que os resultados não possam ser isolados unicamente ao instituto, uma vez que a gestão de risco compete ao conjunto integrado do sistema de controle interno (SCI).

\subsection{Modelo COSO de Gerenciamento de Risco}

Bergamini Junior (2005) recorda que risco é um fato da vida corporativa e que as empresas precisam adotar medidas para geri-los. Essa definição para risco está de acordo com os preceitos orientados pelo Committee of Sponsoring Organizations of the Treadway Commission [COSO] (Dantas, Rodrigues, Marcellindo \& Lustosa, 2010).

A proposta do COSO através do documento Enterprise Risk Management [COSO ERM], estabelece medidas para o tratamento adequado ao risco corporativo, sendo ampla e aplicável às organizações por meio de conceitos importantes de como manter o risco compatível com o apetite da organização.

Dessa maneira, o risco corporativo requer gerenciamento que pressupõe estágios para a administração do evento: a) a identificação do risco pelo qual enfrenta a empresa; b) a medida de impacto potencial do risco identificado; e c) a decisão se cada risco relevante possa ser minimizado (Fama, Cardoso \& Mendonça, 2003).

De acordo com Brito (2007), é a etapa do processo de tratamento aos riscos que se refere à identificação e à avaliação dos eventos considerados inaceitáveis pela organização para adoção das medidas de resposta adequada. Todavia, segundo o COSO ERM (2007), a administração precisa avaliar o efeito sobre a probabilidade de ocorrência e o impacto deste risco sobre a organização.

O COSO ERM se refere a uma adição à estrutura conceitual do COSO Report, passando o controle interno a fazer parte integral do gerenciamento de risco corporativo (CVM, 2015), enfatizando os objetivos estratégicos da corporação, além da ação ampliadora de avaliação de riscos, segundo os quatro subcomponentes: fixação de objetivos (comum à estrutura dos controles internos, excetos pelos objetivos estratégicos), identificação de eventos que resultem em impactos nos objetivos da organização, avaliação dos riscos e resposta aos riscos.

Para o COSO ERM (2007), as atividades de controle são políticas e procedimentos que direcionam as ações individuais na implementação das políticas de gestão de risco, de modo que as respostas selecionadas sejam executadas de forma oportuna.

Com isso, na consideração da resposta mais adequada, a administração precisa avaliar o efeito sobre a probabilidade de ocorrência e o impacto do risco, assim como os custos e benefícios esperados, para que a resposta selecionada mantenha os riscos dentro da tolerância desejada. 


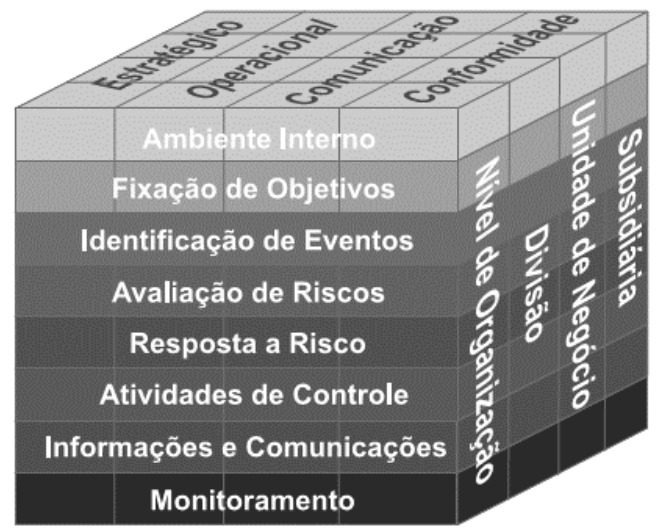

Figura 1 - Matriz COSO ERM

Nesse caso, a probabilidade e o impacto da ocorrência de risco, como fraudes na organização, pode ser reduzida (mitigada), englobando os pilares do modelo estrutural sugerido pelo CO$\mathrm{SO}$, a partir da definição das estratégias (Strategic), com o uso eficaz dos recursos (Operations), para a reprodução dos resultados (Reporting), através do respeito às leis e regulamentos (Compliance) (Dutra, Zanette, Alberton, Bornia, \& Limongi, 2009).

\section{METODOLOGIA}

\subsection{Método}

Este estudo considera o comportamento para compliance um instrumento do processo de gestão no desenvolvimento do ambiente de controle, logo, para que retorne a conclusão de que o compliance representa uma ferramenta de controle interno para mitigação aos riscos, a princípio, parte-se da dedução do que se encontra, até implicitamente, nas premissas consideradas:

a) Premissa $1(\mathrm{P} 1)$ - a conformidade às regras está sob o aparato do sistema de controle interno;

b) Premissa 2 (P2) - o estado de agir em conformidade aos padrões e valores, refere-se ao compliance como parte integrante da cultura organizacional, em menor ou maior intensidade;

c) Premissa $3(\mathrm{P} 3)$ - a gestão de riscos parte de atividades de controle em políticas e procedimentos que direcionam as ações individuais, de modo que as respostas selecionadas aos riscos existentes sejam executadas de forma adequada e oportuna.

Assim, o método adotado neste estudo é o dedutivo, porque diz que os argumentos estão corretos ou incorretos, ou as premissas sustentam de modo completo a conclusão ou, quando a forma é logicamente incorreta, não a sustentam de forma alguma, ou seja, sem graduações intermediárias.

Desse modo, sustentado por Lakatos e Marconi (2003), esse argumento dedutivo reformula ou enuncia de modo explícito a informação que já está contida nas premissas consideradas, segundo as quais, compliance é uma ferramenta do sistema de controle interno, tendo como preponderância a capacidade de resposta ao risco, através do comportamento de conformidade inserido no ambiente de controle. 


\subsection{Tipologia da Pesquisa}

Para atingir o objetivo proposto no estudo é necessário entendê-lo como um evento qualitativo a partir de uma abordagem quantitativa. Pretende-se traduzir em números a percepção do ambiente corporativo para execução da análise dos dados.

Logo, a abordagem metodológica aplicada nesta pesquisa se caracteriza como quantitativa baseada em levantamento de dados através de questionário, cujas perguntas se apresentam objetivas e estruturadas com o uso de escala likert de cinco pontos.

De acordo com Grinnel (1997 como citado em Sampieri, Collado \& Lucio, 2010, p. 4), embora o enfoque quantitativo guarde fases similares como o enfoque qualitativo, a abordagem se vale do levantamento de dados para provar hipóteses baseadas na medida numérica e da análise estatística para montar padrões de comportamento.

$\mathrm{Na}$ condução da pesquisa, foram elencadas duas variáveis consideradas, o Nível de Intensidade Compliance (x), e como variável dependente, a Resposta ao Risco (y).

A abordagem metodológica, nesse caso, dar-se de maneira quantitativa com uso de variáveis definidas como qualitativas, cuja mensuração obedece aos correspondentes atributos/características distribuídos em escala ordinal.

\subsection{Definição das Variáveis: Preparação para as Escalas Compliance e Risco}

As variáveis desta pesquisa são do tipo qualitativas por suas características presentes (atributos), as quais obedecem a uma relação de ordem (variáveis ordinais). E conforme sua natureza não métrica, a mensuração das variáveis escalares ocorre a partir da conversão dos atributos e respectivos pesos obtidos, de modo que a maneira como estas são medidas determina as técnicas estatísticas apropriadas.

$\mathrm{Na}$ intenção de se alcançar uma medida confiável do nível de intensidade compliance associado à capacidade da organização em responder aos riscos (segundo a probabilidade de ocorrência), desenvolve-se um instrumento de coleta capaz de medir a cultura compliance na organização sobre a ação mitigadora da ocorrência dos riscos, mediante a percepção dos sujeitos pesquisados com a utilização de questões, cujas respostas não seriam possíveis obtê-las senão através deste procedimento.

\subsubsection{Medida para Cultura Compliance (Escala NIC)}

A escala do Nível de Intensidade Compliance (NIC) está dividida em duas seções, sendo na primeira seção, os respondentes são instigados a apontar seu entendimento sobre os conceitos e operacionalização do compliance corporativo. Na seção 2, os respondentes são provocados a externar a chance de ocorrência de riscos para a compreensão do nível de controle disseminado na organização que oportunize a mitigação do evento. São os valores e atitudes diante de compliance.

Na seção 1, o instrumento está estruturado, segundo as diretrizes para programas de compliance: Good Practices on Internal Controls, Ethics and Compliance [OCDE]; Resource Guide to the US Foreign Corrupt Practices Act [FCPA]; Six Principles to Prevent Bribery [UK Bribery Act] e o Programa de Integridade da CGU (2015).

Nesta seção, os respondentes são instigados a indicarem o grau de concordância quanto as afirmações constantes sobre os elementos conceituais imprescindíveis para um compliance organizacional em uma escala invertida de 5 pontos: 1 . Concordo Integralmente; 2 . Concordo Parcialmente; 3. Indiferente; 4. Não Concordo Parcialmente; 5. Não Concordo Integralmente; em que, quanto maior a pontuação alcançada, menor o grau de compliance. 
Na seção 2, os respondentes são instigados a cenários éticos questionáveis para se medir o grau de atitude (valores) em relação à compliance individual. Adaptação da Escala de Longenecker et al. (2006), sob a tradução e ajuste dirigidos por Terra (2015).

A abordagem nesta seção compreende a dimensão dos (vi) valores e atitudes diante de compliance, em que se imprime o valor do indivíduo que interfere na organização. A seção está disposta em uma escala de 5 pontos: 1 . Inaceitável; 2. Poucas Vezes Aceitável; 3. Indiferente; 4. Muitas Vezes Aceitável; 5. Totalmente Aceitável, indicando quanto maior o resultado, pior é a percepção dos respondentes em se tratando de compliance.

Por fim, estabelece como critério para avaliar a intensidade compliance, aplicando um escore com base na média de todos os indicadores (atributos) das dimensões de compliance estudadas para a formação da escala NIC.

Quadro 1 - Classificação da escala compliance (NIC)

\begin{tabular}{|c|c|c|}
\hline Escore & Índice $N I C$ & Análise da Frequência \\
\hline 1 & 1 & Alta \\
\hline 2 & $1-2$ & Média \\
\hline 3 & $>2$ & Baixa \\
\hline
\end{tabular}

Fonte: Elaboração própria.

Assim, vislumbra para o primeiro escore (1) um NIC de alta frequência, quando o resultado for igual a 1 no índice da média das respostas da escala, o escore (2) para um NIC de média frequência que varia entre os intervalos 1 - 2 das médias das respostas para o referido grupo e, o escore (3) para um NIC de frequência baixa, quando superar o ponto 2 do intervalo, ou seja, o resultado da média for maior que 2 no índice da escala.

A ideia é classificar a organização com base na estrutura de compliance encontrada, indicando quanto mais próximo de 1 na escala, maior a intensidade compliance.

\subsubsection{Medida para Risco (Escala MIT)}

A disposição da escala de Risco (MIT) está representada na seção 3 do instrumento de coleta. Nesta seção, os respondentes são provocados a externar a chance (probabilidade) de ocorrência de riscos para a compreensão da intensidade de compliance disseminado na organização que oportunize o nível de resposta mitigadora do evento.

A seção se estrutura, conforme os parâmetros do COSO ERM (2007) suportados na perspectiva sobre a avaliação do impacto e probabilidade do evento para determinação da resposta adequada. Também, nas menções de Paulo, Fernandes, Rodrigues e Eidt (2007), segundo a mensuração da probabilidade de ocorrência dos riscos, e, por fim, em orientações decorrentes das pesquisas da KPMG (2015a; 2015b), apresentando o tipo de risco mais relevante para a função de compliance no Brasil.

Espera-se que a escala de risco (MIT) satisfaça razoavelmente a mensuração do risco de fraude e regulatório da organização, ressaltando que a oportunidade de ocorrência faz concluir sobre a eficácia ou não dos controles adotados.

Assim, reconhecendo que não se podem isolar os resultados tão somente ao compliance, por que a gestão de risco compete ao conjunto integrado de atividades de controle (SCI), elegem-se aqueles riscos considerados mais relevantes e de maior impacto para a função de compliance no 
Brasil, segundo a KPMG (2015a): (i) trabalhistas, segurança do trabalho, tributário e previdenciário; (ii) fraudes, como apropriação indébita, e corrupção.

Portanto, a mensuração do risco, segundo a probabilidade de ocorrência, segue recomendação encontrada nos estudos de Paulo et al (2007), quanto à classificação oportuna da probabilidade de ocorrência de risco, considerando o grau de frequência (em escala de 5 pontos): 1 . Muito Raro; 2. Raro; 3. Eventual; 4. Frequente; 5. Muito Frequente.

No processo de avaliação da escala MIT, aplica-se a mesma regra adotada na construção da escala NIC, estabelecendo o índice correspondente à média alcançada das respostas dos indicadores aplicados sobre a estrutura de controle da organização baseado na probabilidade de ocorrência do risco de fraude e regulatório.

Quadro 2 - Classificação da escala de Risco (MIT)

\begin{tabular}{|c|c|c|}
\hline Escore & Índice $M I T$ & Análise da Estrutura de Controle \\
\hline 1 & 1 & Estável \\
\hline 2 & $1-2$ & Regular \\
\hline 3 & $2-3$ & Instável \\
\hline 4 & $3-4$ & Muito Instável \\
\hline 5 & $>4$ & Totalmente Insegura \\
\hline
\end{tabular}

Fonte: Elaboração própria.

O interesse é classificar a organização com base em sua capacidade de responder aos riscos de fraudes e regulatórios, imprimindo quão mais próximo de 1 na escala, melhor o esforço da organização em responder aos eventos.

Dessa forma, o escore (1) é característico para um nível de resposta aceitável ao risco, que não quer dizer "excelente" (devido à força e o impacto do evento que resta total intolerância na chance de sua ocorrência); o escore (2) que faz alusão ao nível de resposta para uma estrutura de controle que já aponta uma segurança regular diante da oportunidade de ocorrência de eventos desastrosos; o escore (3) no nível de resposta, conforme uma estrutura comprometida diante da probabilidade moderada de ocorrência dos riscos na organização; o escore (4) do nível de resposta para uma estrutura fragilizada diante de alta probabilidade de ocorrência do evento na organização, e escore (5) do nível de resposta para ausência total de controle, em que a ocorrência de fraudes e outros riscos serão inevitáveis.

Por conseguinte, o instrumento de coleta se encerra em quatro seções. As duas primeiras abordadas no início desta seção estão formuladas com as questões do tipo Likert (assertivas) para atender a variável independente $(x)$ compliance. A terceira seção contém conjunto de afirmativas para examinar o grau de probabilidade de ocorrência de risco referente à variável dependente (y) resposta ao risco. E, a última seção está formulada para analisar as variáveis demográficas para o perfil do respondente, segundo: gênero, idade, área de atuação profissional e a vinculação a empresas listadas ou não na BM\&FBovespa.

A última seção do questionário está formulada para análise das variáveis demográficas.

\subsection{População e Amostra da Pesquisa}

A amostra da pesquisa está delimitada a partir dos profissionais de empresas que operam no Brasil. Abre-se um recorte específico na população-alvo (G2) para admitir profissionais de empresas de grande porte classificadas pelo faturamento e que estejam listadas na BM\&FBovespa. 
A ideia é comparar o desempenho com o primeiro grupo da amostra (G1) sem recorte específico, analisando o comportamento desses profissionais que trabalham em corporações que, em tese, tem estratégia definida diferenciada de fazer gestão, de estrutura de controles internos e de transparência exigidas para organizações de capital aberto, além de se está diante de corporações de melhor nível de governança corporativa quando se referem a empresas de porte maior (Silveira, 2004).

Para este recorte especial, foram escolhidas empresas de capital aberto que tiveram suas ações ou títulos negociados na Bolsa de Valores em (2016), considerando os segmentos do Novo Mercado e Tradicional e cuja receita bruta de vendas auferida no ano-calendário anterior (dados de 01/01/2015 a 31/12/2015) tenha ultrapassado os R\$ 300 milhões, segundo critério do BNDES para classificar empresas de grande porte.

O resultado da seleção para o grupo G2 compreendeu ao final, 121 empresas aptas para aplicação do questionário de pesquisa, através dos profissionais que aceitarem em participar.

\subsection{Coleta e Tratamento dos Dados}

A pesquisa foi realizada com profissionais de empresas brasileiras, acessados através da rede social profissional Linkedin, redes sociais de relacionamentos em grupos fechados (Facebook e WathsApp), canal disponibilizado pelas corporações, e entrevistas.

Ao todo, foram enviados 943 (novecentos e quarenta e três) questionários distribuídos entre os dois grupos amostrais (G1 e G2) dedicados às diversas atividades corporativas: de monitoramento e fiscalização, financeira e contábil, administrativa e recursos humanos, informática, comercial, vendas e marketing, entre outras.

O interesse é capturar a quem se dedica principalmente na parte operacional, por esperar deles a compreensão mais real possível da vivência e cultura corporativa do que dirigir a pesquisa baseado em opinião de quem somente administra e toma decisões.

A amostra final (n) foi composta por 234 (duzentos e trinta e quatro) profissionais que concluíram o questionário da pesquisa (disponibilizado entre o período de 02/12/2016 a 28/12/2016). Todas as respostas foram utilizadas em sua totalidade, denotando que o tamanho da amostra consta do total de registros obtidos $(n=234)$, sendo o tamanho da amostra de 135 para o grupo $(\mathrm{G} 1=$ 135) e 99 para o grupo (G2 = 99).

Antes da aplicação do questionário, procedeu-se a dois pré-testes com um pequeno grupo semelhante à amostra, cujo objetivo vislumbrou apurar a validade do instrumento de coleta, verificando a objetividade e compreensibilidade das questões elaboradas sob o exame da aplicabilidade funcional e prática do questionário na intenção de se afastarem erros de interpretação e confusão no contato com as perguntas.

Para o tratamento dos dados, utilizaram-se as técnicas de inferência com teste de MannWhitney para comparabilidade entre os grupos da amostra, teste descritivo por meio da distribuição de frequência com a análise do desempenho dos grupos amostrais por escala avaliada e o uso da técnica multivariada de análise fatorial (KMO) e teste de Alfa de Cronbach para a validade e consistência interna das escalas.

Por fim, o processamento dos dados ocorreu por meio do software IBM SPSS Statistics 22, mediante a extração das respostas válidas obtidas pelo formulário do Google Form. 


\section{APRESENTAÇÃO E ANÁLISE DOS RESULTADOS}

Nesta seção, são apresentadas a análise e a descrição dos dados coletados na aplicação da pesquisa, de acordo com a sequência: a) participantes; b) análise da validade e confiabilidade do instrumento; c) análise do comportamento da amostra.

\subsection{Participantes}

A característica e perfil dos participantes configuram um dos pontos fortes desta pesquisa. Os respondentes são de todas as regiões do Brasil com profissionais de diversas áreas, idades e vinculados a empresas de diferentes tamanhos e tipos de negócio.

Na distribuição dos dados por grupo amostral, prevalece, porém de maneira equilibrada, a participação do público feminino nos dois grupos amostrais, com 51,1\% para o grupo G1 e 50,5\% da participação para o grupo G2. Em relação à idade dos respondentes, a maior parte deles se concentram entre 21 a 40 anos de idade, compreendendo 71,1\% para o grupo G1 e 77,8\% para o grupo G2.

Tabela 1: Perfil dos Respondentes

\begin{tabular}{|c|c|c|c|c|c|}
\hline \multirow{2}{*}{\multicolumn{2}{|c|}{ Variáveis Demográficas }} & \multicolumn{2}{|c|}{ G1 } & \multicolumn{2}{|c|}{ G2 } \\
\hline & & $\mathbf{N}$ & $\%$ & $\mathbf{N}$ & $\%$ \\
\hline \multirow{3}{*}{ Gênero } & Feminino & 69 & 51,1 & 49 & 50,5 \\
\hline & Masculino & 66 & 48,9 & 50 & 49,5 \\
\hline & & 135 & 100,0 & 99 & 100,0 \\
\hline \multirow{6}{*}{ Idade } & 21 a 30 & 49 & 36,3 & 40 & 40,4 \\
\hline & 31 a 40 & 47 & 34,8 & 37 & 37,4 \\
\hline & 41 a 50 & 25 & 18,5 & 16 & 16,2 \\
\hline & 51 a 60 & 13 & 9,6 & 6 & 6,1 \\
\hline & 61 a 70 & 1 & 0,7 & & \\
\hline & Maior que 70 & & & & \\
\hline \multirow{6}{*}{ Área de Atuação } & & 135 & 100,0 & 99 & 100,0 \\
\hline & Administrativa/RH & 22 & 16,3 & 20 & 20,2 \\
\hline & Financeira/Contábil & 23 & 17,0 & 21 & 21,2 \\
\hline & Comercial/Vendas/Marketing & 15 & 11,1 & 15 & 15,2 \\
\hline & Informática/TI & 8 & 5,9 & 6 & 6,1 \\
\hline & Fiscalização/Monitoramento & 47 & 34,8 & 25 & 25,3 \\
\hline \multirow{7}{*}{ Listada Bovespa } & Outras & 20 & 14,8 & 12 & 12,1 \\
\hline & & 135 & 100,0 & 99 & 100,0 \\
\hline & Tradicional & & & 66 & 66,7 \\
\hline & Novo Mercado & & & 33 & 33,3 \\
\hline & Outro Segmento & & & & \\
\hline & & & & 99 & 100,0 \\
\hline & Não Listada Bovespa & 135 & 100,0 & & \\
\hline
\end{tabular}

Fonte: Elaboração própria.

Destacando a característica da amostra, os dados se distribuem em 135 (=58\%) registros de respondentes pertencentes ao grupo G1 de profissionais de empresas sem recorte, enquanto que 99 $(=42 \%)$ dos registros de participantes pertencem ao grupo G2 de profissionais de empresas listadas na Bovespa consideradas de grande porte. A respeito destas empresas do grupo G2, 66,7\% se referem ao segmento Tradicional, e 33,3\% ao segmento do Novo Mercado Bovespa. 


\subsection{Validade e Confiabilidade do Instrumento}

Esta seção confirma a aplicabilidade do questionário sobre os conhecimentos teóricos e operacionais de compliance, bem como as habilidades organizacionais para o enfrentamento aos riscos decorrentes de fraudes e regulatórios com base na probabilidade de ocorrência do evento.

Para determinar a validade do instrumento de escala NIC, procedeu-se a análise fatorial dos dados com a intenção de identificar os fatores comuns na relação entre as variáveis tratadas. $\mathrm{O}$ resultado KMO na análise da escala NIC com os 11 itens (atributos) avaliados destinados à medição da intensidade compliance foi de 0,79 para dois fatores considerados, sendo o primeiro de caráter positivo à medida que o assunto explorado busca a aplicação de conceitos de compliance e o segundo fator explicando o aspecto negativo por sugerir temas relacionados a condutas de não conformidade em ambiente corporativo. $\mathrm{Na}$ análise dos componentes principais, foram retidos dois fatores com autovalor superior a 1 que explicam cerca de 49,74\% de variabilidade total.

O teste de esfericidade de Bartlett para a escala NIC foi significativo (Sig<0,05), demonstrando que as variáveis estão correlacionadas significativamente e a matriz de identidade é favorável à análise.

Tabela 2 - Resultado do teste de validade KMO e Bartlett para escala compliance (NIC)

\begin{tabular}{llc}
\hline \multicolumn{2}{l}{ Medida Kaiser-Meyer-Olkin de adequação de amostragem } &, 79 \\
\hline \multirow{3}{*}{ Teste de esfericidade de Bartlett } & Aprox. Qui-quadrado & 643,860 \\
& Df & 55 \\
& Sig. &, 000 \\
\hline
\end{tabular}

Fonte: Elaboração própria

O resultado do teste $\mathrm{KMO}$ confirma para uma adequação a nível moderado sobre os dados obtidos no tratamento através da técnica de análise fatorial (com índice 0,7 a 0,8), convencendo-se a partir de Favero, Belfiore, Siva e Chan (2009), de quanto mais próximos de 1, mais adequada é a utilização da técnica. A técnica KMO avalia a adequação da amostra em relação à técnica fatorial.

A interpretação definida pelo resultado das cargas fatoriais após rotação dos fatores pelo método oblimin na identificação da cultura de compliance mensura para o Fator 1: a força (intensidade) sobre as práticas organizacionais que se voltam a construção ideal de um ambiente de conformidade (aspecto positivo), enquanto que o Fator 2: a força desses valores medidos sobre o comportamento do indivíduo diante de problemas de não conformidade (aspecto negativo).

A adequação orientada nos resultados dos testes KMO com variação dos dados em 49,74\% e uma significância para o teste de esfericidade de Bartlet se preserva a todos os atributos da escala, inclusive aqueles de menor carga apresentado pelo teste, resultando válido o instrumento.

Para determinação do nível de confiabilidade da escala NIC, procedeu-se ao coeficiente $\boldsymbol{\alpha}$ de Cronbach suficiente para encontrar uma estimativa da confiabilidade do questionário aplicado, dado que todos os itens do instrumento de coleta utilizam a mesma escala de medição, sendo o Alfa calculado a partir da variância dos itens individuais e das covariâncias entre os itens para avaliar a consistência da escala inteira.

O resultado do teste indicou um nível aceitável de confiabilidade do constructo em vista do Alfa de Cronbach superior a 0,7 (Alfa igual a 0,74). Com isto, pode-se inferir como boa fiabilidade da escala construída. 
Tabela 3 - Resultado do teste de confiabilidade Alfa de Cronbach para escala compliance (NIC)

Alfa de Cronbach Alfa de Cronbach com base em $\quad$ N. de itens

itens padronizados

,74 74

Fonte: Elaboração própria

Confirma a consistência interna com base no Alfa igual a 0,70 a 0,82 esperado para as ciências sociais e humanas (MOROCO; GARCIA-MARQUES, 2006).

Em relação à determinação da validade e confiabilidade da segunda escala proposta na pesquisa, escala de risco (MIT), procedeu-se a análise fatorial dos dados com a intenção de identificar os fatores comuns na relação com as variáveis tratadas. O resultado do teste fatorial representou na medida de adequação da amostragem considerada alta com KMO próximo de 0,9 $(K M O=0,87)$ para um único fator com variância explicada de $44,31 \%$.

O teste de esfericidade de Bartlett para a escala foi significativo (Sig $<0,05)$, demonstrando que as variáveis estão correlacionadas significativamente e a matriz de identidade é favorável à análise.

Tabela 4 - Resultado do teste de validade KMO e Bartlett para escala ao risco (MIT)

\begin{tabular}{|c|c|c|}
\hline \multicolumn{2}{|c|}{ Medida Kaiser-Meyer-Olkin de adequação de amostragem } & 87, \\
\hline \multirow{3}{*}{ Teste de esfericidade de Bartlett } & Aprox. Qui-quadrado & 650,781 \\
\hline & Df & 36 \\
\hline & Sig. & 000, \\
\hline
\end{tabular}

Fonte: Elaboração própria.

Os itens do questionário apresentam uma carga fatorial com poder de explicação com base no próprio modelo executado. Logo, a escala se encontra válida pelo teste KMO obtido com uma significância estatística na aplicabilidade.

O procedimento seguinte sucedeu ao teste de confiabilidade do constructo para reconhecer se a escala tratada tem fidedignidade na precisão de seus itens. O coeficiente Alfa de Cronbach obtido superior a 0,8 indica uma estimativa com boa relação interna de consistência entre os itens do questionário aplicado (Alfa de Cronbach $=0,84$ ).

Tabela 5 - Resultado do teste de confiabilidade Alfa de Cronbach para escala de risco (MIT)

Alfa de Cronbach Alfa de Cronbach com base em N. de itens

itens padronizados

,84 84

Fonte: Elaboração própria

Portanto, respeitando os métodos adotados na análise, preserva-se o instrumento na sua integralidade conforme a abrangência estatística, demonstrando aceitável a confiabilidade do constructo para o Alfa de Cronbach igual a 0,74 em relação à escala NIC e Alfa de 0,84 relacionado à escala MIT.

\subsection{Análise do Comportamento da Amostra}

O objetivo da seção contempla a intenção de analisar o comportamento dos grupos amostrais diante das práticas difundidas para compliance e a eficácia para resposta adequada aos riscos de fraude e regulatório. 
No primeiro teste realizado referente a distribuição de frequência sobre o desempenho do grupo G1 para as escalas compliance (NIC) e risco (MIT), vislumbra que a amostra com 135 participantes tem distribuição dos dados agrupados assumindo dois intervalos para a escala NIC e quatro intervalos para a escala MIT, sendo na primeira escala um valor mínimo obtido de 1 e máximo para 3 e, para a segunda escala, mínimo de 1 e máximo de 5 na escala definida.

Tabela 6 - Comportamento do grupo (G1) com base na distribuição da escala compliance (NIC)

\begin{tabular}{lcccc}
\hline Escala NIC & Frequência & Relativa $(\%)$ & Média & DP \\
\hline Alta intensidade compliance & 5 & 3,7 & 1,73 & 0,47 \\
Média intensidade compliance & 94 & 69,6 & & \\
Baixa intensidade compliance & 36 & 26,7 & & \\
Total & 135 & 100,0 & & \\
\hline
\end{tabular}

Fonte: Elaboração própria.

Na distribuição dos dados para o tamanho amostral obtido do grupo G1 ( $\mathrm{n}=135)$, verificou-se que no comportamento dos dados, mais de $69 \%$ concentram-se na intensidade média de compliance (escore 2 da escala), enquanto que pouco mais de $3 \%$ da distribuição dos dados obtidos estão no escore máximo (1) de intensidade alta compliance. No resultado, $27 \%$ das médias dos registros totais distribuem-se na intensidade baixa compliance (escore 3).

Tabela 7 - Comportamento do grupo (G1) com base na distribuição da escala risco (MIT)

\begin{tabular}{lcccc}
\hline Escala MIT & Frequência & Relativa $(\%)$ & Média & DP \\
\hline Estável & 3 & 2,2 & 2,25 & 0,73 \\
Regular & 53 & 39,3 & & \\
Instável & 61 & 45,2 & & \\
Muito instável & 15 & 11,1 & & \\
Totalmente insegura & 3 & 2,2 & & \\
Total & 135 & 100,0 & & \\
\hline
\end{tabular}

Fonte: Elaboração própria.

$\mathrm{Na}$ análise da distribuição dos dados para a escala MIT de risco, tem-se que, tão somente, $41,5 \%$ da amostra indicam capacidade e controle em responder aos riscos avaliados, contra 58,5\% que demonstram níveis inaceitáveis de resposta aos riscos investigados, de acordo com a alta probabilidade de ocorrência dos eventos.

Na análise da distribuição dos dados, $11 \%$ da amostra se colocam como muito instável na capacidade de resposta com forte probabilidade de ocorrência de fraudes e regulatórios, enquanto que outra parcela da amostra $(2,2 \%)$ se mostra totalmente vulnerável com riscos a níveis inaceitáveis, cuja ocorrência se dá de maneira inevitável na organização, conforme a percepção dos trabalhadores da amostra.

Para determinar o desempenho do grupo G2 no tocante às escalas compliance (NIC) e risco $(M I T)$, o teste de distribuição de frequência vai compreender uma amostra de tamanho de 99 participantes, cuja distribuição dos dados agrupados assumem dois intervalos para a escala NIC e três intervalos para a escala MIT, sendo o desempenho da primeira escala um valor mínimo obtido igual a 1 e máximo para 3 e, para a segunda escala, desempenho igual a 1 na escala e máximo de 4. 
Tabela 8 - Comportamento do grupo (G2) com base na distribuição do escore compliance (NIC)

\begin{tabular}{lcccc}
\hline Escala NIC & Frequência & Relativa $\%$ & Média & DP \\
\hline Alta intensidade compliance & 9 & 9,1 & 1,87 & 0,55 \\
Média intensidade compliance & 53 & 53,5 & & \\
Baixa intensidade compliance & 37 & 37,4 & & \\
Total & 99 & 100,0 & & \\
\hline
\end{tabular}

Fonte: Elaboração própria.

$\mathrm{Na}$ análise do desempenho para escala compliance (NIC), a maior frequência verificada entre as médias dos registros por atributo se concentrou na média de intensidade compliance (escore 2) com um percentual de $54 \%$, e apenas $9 \%$ com alta frequência de compliance (escore 1). No resultado dos registros verificados (média consolidada) para os atributos NIC, 37\% correspondem a baixa intensidade compliance (escore 3).

Um grau de intensidade abaixo do esperado, considerando a característica da amostra (empresas de grande porte listada na Bovespa, segundo os níveis Tradicional e Novo Mercado). O mesmo comportamento se verifica na análise das frequências dos registros tomados para a escala de risco (MIT), vide Tabela 8, em que 61 \% dos registros apontam para um nível de tolerância ao risco capaz de demonstrar incapacidade de toda a estrutura de controle para resposta à ocorrência de fraudes e riscos regulatórios.

Tabela 9 - Comportamento do grupo (G2) com base na distribuição da escala de risco (MIT)

\begin{tabular}{lcccc}
\hline Escala MIT & Frequência & Relativa $(\%)$ & Média & DP \\
\hline Estável & 3 & 3,0 & 2,36 & 0,59 \\
Regular & 25 & 25,3 & & \\
Instável & 61 & 61,6 & & \\
Muito instável & 10 & 10,1 & & \\
Total & 99 & 100,0 & & \\
\hline
\end{tabular}

Fonte: Elaboração própria.

Dos resultados extraídos, apenas $3 \%$ dos registros se encontram com grau de segurança aceitável como resposta mitigadora aos riscos avaliados, tendo em vista a pequena chance de eventos fraudulentos e regulatórios acontecerem.

Porém, isto representa que $71 \%$ dos dados da amostra (segundo o comportamento dos registros médios) não têm condições de responder adequadamente às fraudes e aos riscos regulatórios, encontrando-se em um nível predisposto à ocorrência dos eventos, contra $28,3 \%$ da amostra total, sendo 3\% desta com a capacidade aceitável e em condições de responder adequadamente aos riscos.

Com a análise sobre o comportamento individual, é prematuro ainda afirmar a hipótese de que uma amostra apresenta ou não melhor desempenho em relação a outra. Para isto, aplica-se o teste U de Mann-Whitney para comparar os resultados entre os grupos amostrais e saber se existem igualdade ou não entre eles e qual a força que impacta na consideração desse resultado.

Contudo, esperava-se que os resultados gerais obtidos com a distribuição da frequência dos dados sobre o comportamento de cada amostra pesquisada, especialmente para o grupo (G2), tivesse uma performance mais centrada na política de comprometimento e valores à disposição da estrutura organizacional, haja vista o impacto dos riscos gerados para a corporação. No entanto, os 
esforços da organização provados a partir da capacidade de resposta aos eventos apresentam demonstrações de limitações nos controles e estratégia aplicáveis na transformação da cultura organizacional segundo dilemas éticos e de conformidade.

Procedendo ao teste de comparabilidade entre os grupos da amostra da pesquisa, o resultado do teste de Mann-Whitney demonstra a existência de diferença estatisticamente significativa ( $\mathrm{p}$ $<0,05)$ entre os grupos da amostra em relação a escala de intensidade compliance (NIC), configurando para uma proporção (tendência) maior de cultura de conformidade disseminada pela amostra (G2) na comparação entre as medianas obtidas.

Já na comparação para a escala de risco (MIT), o resultado do teste (U) pondera para uma igualdade estatisticamente aferida entre as amostras. Isto é, do ponto de vista estatístico não há diferença significativa de comportamento entre os grupos (G1) e (G2) da amostra para um p-value igual a ,141.

Tabela 10 - Teste U de Mann-Whitney na comparação entre os grupos da amostra

\begin{tabular}{cccccc}
\hline & $\begin{array}{c}\text { Amostras (Pro- } \\
\text { fissionais) }\end{array}$ & $N$ & Mediana & Postos da Média & Mann-Whitney \\
& G1 & 135 & $\mathbf{1 , 6 3 6 4}$ & $\mathbf{1 1 0 , 0 0}$ & U \\
\multirow{2}{*}{ Escala NIC } & $G 2$ & 99 & $\mathbf{1 , 8 1 8 2}$ & $\mathbf{1 2 7 , 7 2}$ & $\mathbf{0 , 4 8}$ \\
& $G 1$ & 135 & 2,2222 & $\mathbf{1 1 1 , 9 3}$ & \multirow{2}{*}{ NS } \\
\hline
\end{tabular}

Fonte: Elaboração própria.

Nota: NS - Não há significância.

Subsidiariamente, complementa a questão da pesquisa sobre a análise do comportamento da amostra, em que NÃO se pode afirmar que o grupo G2 possui melhor desempenho na efetivação e estrutura de combate às fraudes e riscos regulatórios em relação ao grupo G1. Esta afirmação considera, além do desempenho de cada amostra no teste de distribuição dos dados, o resultado do teste não paramétrico de Mann-Whitney, em que não se verificaram diferenças estatisticamente significativas $(\mathrm{p}=, 121)$ entre as amostras para a escala MIT.

Com isso, apesar de não rejeitar o pressuposto da pesquisa, respeitando as limitações deste estudo e concordando que esta não é a única variável que mantém relação de influência com a variável dependente para o risco, os achados são sutis, inferindo que esforços de difusão para uma atividade efetiva de compliance no Brasil ainda se disponham incipientes, logo, por vezes controversos.

De maneira geral, os resultados da pesquisa demonstram certa incoerência, mas acompanham a interpretação de Perera, De Freitas e Imoniana (2014) de que a atividade de compliance, mesmo com a alta avaliação de eficácia da área de compliance no combate às fraudes que a torna um dos principais componentes do sistema de controle interno, não exime a empresa de atos fraudulentos.

Contudo, atenta-se para o emprego extrínseco da propulsão de compliance protocolizada, sem considerar a definição de valores organizacionais e de comprometimento ético, conforme criticado por Wellner (2005) e Stucke (2013) sobre as distorções criadas pelo modelo americano para compliance program, na medida em que (i) encorajam as corporações a adotarem programas subotimos, (ii) privilegiam executivos da alta hierarquia, e (iii) desencorajam pequenas e médias empresas devido ao custo de implementação do programa. 
A consequência, para tanto, traduz-se na formação de estrutura de controle organizacional extremamente frágil, baseada em reação compulsória, não transformadora para uma "cultura" de integridade, levando a corporação a considerar exclusivamente a formalidade prescrita (Porto; Tamayo, 2005).

Daí a compreensão mais sensata, conforme Migliavacca (2002), o de que a ocorrência de fraudes e outros tipos de riscos relacionados à compliance decorre da negligência de mecanismos eficazes de governança.

\section{CONSIDERAÇÕES FINAIS}

Estudos recentes justificam a expressão compliance como uma das principais soluções estratégicas disponíveis pela organização para a inibição à ocorrência de riscos (Costa, 2012). O termo tem sido utilizado, inclusive, para transpassar a ideia de comprometimento organizacional.

A discussão sobre as práticas de compliance no Brasil passa a novo patamar com a implementação da Lei Anticorrupção, levando esta ferramenta da gestão de risco, como um instituto compulsório, pois, enquanto neste se baseia a política de formalidade, aquela traz em sua definição a internalização de valores pessoais e organizacionais para a formação de "cultura" de integridade.

É certo que a necessidade de discussão sobre compliance e outros instrumentos de controle sempre estiveram atrelados aos impactos gerados nas organizações diante de ações antiéticas e falhas operacionais que ameaçam a estrutura organizacional como um todo, com grandes somas dos recursos patrimoniais, além do prejuízo da imagem a que se submetem.

Segundo a KPMG (2015a; 2015b), a exposição aos riscos corporativos associados a falhas nos processos regulatórios internos e fraudes é responsável pela maior parte das incertezas no mercado.

A resposta a esses estímulos também tem se repercutido em esforços de organismos internacionais para combate às práticas antiéticas e não regulatórias no universo empresarial com surtido efeito, principalmente, diante das diversas notícias de desmonte de esquemas de corrupção orquestrados por empresários, funcionários e terceiros ligados à atividade econômica e de estado com a cominação de multas e condenações de prisão.

Institutos regulatórios anticorrupção como FCPA e Anti Bribery foram responsáveis pela alavancagem do compliance nas organizações (Trapp, 2015). No Brasil, o destaque para a Lei 12.846/2013, denominada Lei Anticorrupção, que incorpora a figura do compliance ao meio jurídico de forma concreta e compulsória, impondo a necessidade da inclusão de programas de integridade através de incentivos extrínsecos às empresas.

Isto se dá pela necessidade de se vincular nas corporações a excelência pela prática de controle que tenha sua eficácia medida através da confiabilidade das operações, de modo a promover a diminuição das incertezas em relação à sensibilidade a eventos desastrosos futuros. Nisto, os esforços de compliance são difundidos como ferramental capaz de mitigar a ocorrência dos atos danosos (Mazzola, 2015; Costa, 2012).

Nesse contexto, as empresas brasileiras perceberam essa premência de intensificação na estrutura dos sistemas de controle para afastar de seu ambiente ameaças de perda, imposições legais, e, sobretudo, de exposição da imagem. Os dados são da pesquisa realizada pela Deloitte (2015) que revela, entre 2013 e 2015, o crescimento de 100\% no número de empresas que afirmam possuir um programa de compliance efetivo. A Lei Anticorrupção foi um propulsor para a transformação desta realidade no País. 
Esta foi a questão suscitada neste estudo que investigou a relação entre as práticas de compliance e sua eficácia na resposta aos riscos corporativos no Brasil.

Baseado no instrumento validado e consistente, capturou-se a percepção dos respondentes, quanto a cultura de compliance da organização e a capacidade destas corporações em responder aos riscos de fraudes e regulatórios.

Em outras palavras, ao reconhecer compliance uma ferramenta estratégica à disposição da organização (Costa, 2012), buscou-se analisar se, na realidade corporativa, este instituto tem, de fato, impactado na redução dos riscos corporativos.

A análise dos resultados sobre o desempenho dos grupos amostrais para as escalas compliance (NIC) e risco (MIT) demonstra que as empresas brasileiras, especialmente, empresas de grande porte Bovespa (G2), estão aquém do esperado, considerando a qualidade desta amostra e impacto gerado pelos riscos investigados.

Ao proceder testes de comparabilidade sobre o comportamento dos grupos amostrais, o resultado (U) indica a existência de diferença significativa entre a população na intensidade da cultura de compliance (NIC). Mas, do ponto de vista estatístico não há diferenças significativas de comportamento entre os grupos para a escala de risco (MIT).

Apesar de não rejeitar a assertiva de que compliance é um instrumento de mitigação ao risco, os achados podem ser considerados sutis na análise do desempenho dos grupos por escala e de comparabilidade entre a população examinada, sugerindo que os esforços para o efetivo compliance no Brasil para a transformação da cultura organizacional ainda sejam incipientes.

A conclusão da pesquisa confirmou que compliance representa uma ferramenta preponderante no processo de mitigação ao risco, ainda que o resultado tenha demonstrado uma magnitude fraca a moderada na relação entre as variáveis estudadas com base na amostra da pesquisa.

Os achados denotam uma contrariedade da força com que o tema compliance se apresenta no meio corporativo nacional de que ferramentas deste instituto são promotoras reais de combate às fraudes. Confirma, porém, resultados de outros estudos sobre a propensão corruptível, quando se verifica a falta de conformidade da organização (Damania, Fredrikisson \& Mani, 2004; Sundstrom, 2012).

A explicação com a pesquisa demonstra que a baixa eficácia de compliance na resposta ao risco de atos ilegais ou antiéticos pode estar relacionada na pressuposição deste tema, o qual considera compliance compulsado a condição de formalidade prescrita, impressa através de estímulos legais, corroborando com os argumentos de Katz e Kahn (1974 como citado em Porto \& Tamayo, 2005), de que organizações pautadas exclusivamente em conformidades regulatórias possuem uma estrutura organizacional extremamente frágil.

Contudo, assim como em todos os estudos dentro das Ciências Sociais, a limitação desta pesquisa cabe ao objeto avaliado que envolve julgamento perceptivo do indivíduo diante de questões relacionadas a valores individuais e organizacionais, o que, por si, pode demonstrar um fator restritivo na generalização de seus resultados.

Por tudo visto, é de certo coerente que sem o fomento da "cultura" de compliance, baseada no fortalecimento ético (Wellner, 2005), até programas de compliance dificilmente obterão êxito (Coimbra \& Manzi, 2010). 


\section{REFERÊNCIAS}

Associação Brasileira de Normas Técnicas. NBR ISO 31000. (2009). Gestão de Riscos - Princípios e diretrizes. Rio de Janeiro.

Associação Brasileira dos Bancos Internacionais; Federação Brasileira de Bancos. (2009). Cartilha Função de Compliance. Recuperado em 14 janeiro, 2017, de http://www.abbi.com.br/.

Ayres, C. H. S. (2014). Programas de Compliance no âmbito da Lei no 12.846/2013: importância e principais elementos. Revista do Advogado, São Paulo, 125 (1), p.42-50.

Bergamini Junior, S. (2005). Controles Internos como um Instrumento de Governança Corporativa. Revista do BNDES: Rio de Janeiro, 12(24), p. 149-188.

BM\&FBOVESPA. Bolsa de Valores de São Paulo. Recuperado em 13 dezembro, 2016, de http://www.bmfbovespa.com.br.

Lei 12.846, de 1ํo agosto de 2013 (2013). Dispõe sobre a responsabilização administrativa e civil de pessoas jurídicas pela prática de atos contra a administração pública, nacional ou estrangeira, e dá outras providências. Diário Oficial da União. Brasília, DF.

Brito, O. (2007). Gestão de riscos - Uma abordagem orientada a Riscos Operacionais. São Paulo: Editora Saraiva.

Cherman, A. \& Tomei, P.A. (2005). Códigos de ética corporativa e a tomada de decisão ética: instrumentos de gestão e orientação de valores organizacionais? Revista de Administração Contemporânea, 9 (3), p. 99-120.

Coimbra, M.A. \& Manzi, V.A. (2010). Manual de Compliance: preservando a boa governança e a integridade das organizações. São Paulo: Atlas.

Comissão de Valores Mobiliários. (2002). Cartilha de Recomendações da CVM sobre Governança Corporativa. Recuperado em 26 junho, 2015, de http://www.ibgc.org.br/userfiles/cartilhacvm.pdf.

Comissão de Valores Mobiliários (2015). Gerenciamento de riscos corporativos: uma análise das diretrizes e das práticas. Recuperado em 14 novembro, 2016, de http://www.cvm.gov.br/export/sites/cvm/menu/acesso_informacao/serieshist/estudos/anexos/Ger enciamento-de-riscos_final_151015.pdf.

Committee of Sponsoring Organizations of the Treadway Commission. (2007). Enterprise risk management. Tradução da versão original. Recuperado em 25 outubro, 2016, de http://www.coso.org.

Committee of Sponsoring Organizations of the Treadway Commission. (2013). Internal control integrated framework. Recuperado em 25 outubro, 2016, de http://www.coso.org.

Controladoria-Geral da União (2015). Cartilha de integridade para pequenos negócios. Recuperado em 24 outubro, 2016, de http://www.cgu.gov.br/publicacoes/etica-eintegridade/arquivos/integridade-para-pequenos-negocios.pdf.

Controladoria-Geral da União. (2015). Guia de implantação de programa de integridade - diretrizes para empresas privadas. Recuperado em 24 outubro, 2016, de 
http://www.cgu.gov.br/publicacoes/etica-e-integridade/arquivos/programa-de-integridadediretrizes-para-empresas-privadas.pdf.

Controladoria-Geral da União. (2016). Programa empresa Pro-ética. Recuperado em 24 outubro, 2016, de http://www.cgu.gov.br/assuntos/etica-e-integridade/empresa-pro-etica.

Costa, S.C. (2012). O compliance como um novo modelo de negócio nas sociedades empresárias. Revista Científica da Faculdade Darcy Ribeiro, (03).

Cunha, L. M. A. (2007). Modelos rasch e escalas de likert e thurstone na medição de atitudes. Dissertação (mestrado em probabilidades e estatística). Departamento de estatística e investigação operacional da Universidade de Lisboa, Portugal. Recuperado em 16 dezembro, 2017, de http://repositorio.ul.pt/bitstream/10451/1229/1/18914_ulfc072532_tm.pdf.

Damania, R., Fredriksson, P, G. \& Mani, M. (2004). The persistence of corruption and regulatory compliance failures: theory and evidence. Public Choice, 12, pp. 363-390.

Dantas, J.A., Rodrigues, F.F., Marcellino, G. F., \& Lustosa, P.R.B. (2010). Custo-benefício do controle: proposta de um método para avaliação com base no coso. Revista Contabilidade, Gestão e Governança, Brasília: 13 (2), p.3-19.

Deloitte. Governance, risk and compliance within any organization is critical. Recuperado em 10 setembro, 2016, de http://www2.deloitte.com/br/pt/pages/risk/topics/governance-risk-andcompliance.html.

Famá, R., Cardoso, R. L., \& Mendonça, O. (2003). Riscos financeiros e não financeiros: uma proposta de modelo para finanças. Research Paper 1(492).

Farias, R. P., De Luca, M. M. M., \& Machado, M. V. V. (2009). A metodologia COSO como ferramenta de gerenciamento dos controles internos. Revista Contabilidade, Gestão e Governança. Brasília: 12(3), p.55-77.

Favero, L. P., Belfiore, P., Siva, F. L., \& Chan, B. L. (2009). Análise dos dados: modelagem multivariada para tomada de decisões. Rio de Janeiro: Elsevier.

Figueiredo, R. S. (2015). Direito de intervenção e lei 12.846/2013: a adoção do compliance como excludente de responsabilidade. 229 f. Dissertação (mestrado em direito público). Programa de pós-graduação em direito público, universidade federal da bahia. Bahia: salvador. 2015.

KPMG. Cutting Through Complexity. (2015a). Pesquisa maturidade do compliance no Brasil. Recuperado em 24 fevereiro, 2017, de https://www.kpmg.com/br/pt/estudos_analises/artigosepublicacoes/documents/advisory/pesquisacompliance-no-brasil.pdf.

KPMG. Cutting Through Complexity. (2015b). Pesquisa ambiente regulatório. Recuperado em 16 fevereiro, 2017, de http://www.kpmg.com/br/pt/estudos_analises/artigosepublicacoes/paginas/pesquisa-ambienteregulatorio-2015.aspx. 
KPMG. Cutting Khrough Complexity. (2009). A fraude no brasil. Relatório de pesquisa. Recupera$\begin{array}{lllll}\text { do } & 16 & \text { fevereiro, } & \text { de }\end{array}$ http://www.kpmg.com.br/publicacoes/forensic/fraudes_2009_port.pdf.

Lakatos, E.M.A; \& Marconi, M. A. (2010). Metodologia Científica. 5 ed. São Paulo: atlas.

Laruccia, M.M; \& Yamada, K.J (2011). O desenvolvimento sustentável e a gestão de compliance em instituições financeiras. Revista Estratégica, 11.

Longenecker, J. G., Moore, C.W., Petty, J.W., Palich, L.E, \& Mckinney, J.A. (2006). Ethical attitudes in small businesses and large corporations: theory and empirical findings from a tracking study spanning three decades. Journal of Small Business Management, 44 (2), p. 167-183.

Maldonado, J., Aquino, M.G., Lofrano, M. L., De Araújo, G.S., \& Tondo, C. (2014) Valores e atitudes diante de compliance. Revista Jovens Pesquisadores, 11 (1).

Maroco, J.; \& Garcia-Marques, T. (2006). Qual a fiabilidade do alfa de cronbach? Questões antigas e soluções modernas? Laboratório de Psicologia, 4 (1), p. 65-90.

Marshall, C. (2002). Medindo e gerenciando riscos operacionais em instituições financeiras. São Paulo: Qualitymark, 480.

Mazzola, C.M.S. (2015). Aplicabilidade das práticas de compliance e controle interno no combate às fraudes corporativas no Brasil. 100f. Dissertação (mestrado em controladoria empresarial). Universidade Presbiteriana Mackenzie, São Paulo.

Paulo, W.L., Fernandes, F.C., Rodrigues, L, G.B., \& Eidt, J. (2007). Riscos e controles internos: uma metodologia de mensuração dos níveis de controle de riscos empresariais. Revista de Contabilidade e Finanças, 43, p. 49-60.

Perera, L.CJ. De Freitas, E.C, \& Imoniana, J. O (2014). Avaliação do sistema de combate às fraudes corporativas no brasil. Revista Contemporânea de Contabilidade, 11 (23), p. 03-30.

Porto, J. B; \& Tamayo, A. (2005). Valores organizacionais e civismo nas organizações. Revista de Administração Contemporânea, 9 (1), p. 35-52.

Sampieri, R. H, Collado, C. F., \& Lucio, P, B. (2010). Metodología de la investigación. 5. Ed. México: Mcgraw Hill.

Silveira, A. M. (2004). Governança corporativa e estrutura de propriedade: determinantes e relação com o desempenho das empresas no Brasil. 250 f. Tese (doutorado em administração). Departamento de administração da faculdade de economia, administração e contabilidade da universidade de São Paulo, São Paulo.

Stucke, M.E (2013). In search of effective ethics \& compliance programs. Recuperado em 16 novembro, 2016, de https://www.researchgate.net/profile/maurice_stucke/publication/259230808_in_search_of_effecti ve_ethics_compliance_programs/links/02e7e52a876359c90b000000.pdf.

Sundstrom, A. (2012). Corruption and regulatory compliance: experimental findings from south african small-scale fisheries. Marine Policy, 36, p. 1255-1264. 
Tamayo, A. (2008). Valores organizacionais e comprometimento afetivo. Revista de Administração Mackenzie, 6 (3), p. 192-213.

Tamayo, A., \& Gondim, M.G.C (1996). Escala de valores organizacionais. Revista de Administração, são Paulo: 31 (2), p. 62-72.

Tarantino, A. (2008). Governance, risk, and compliance handbook: technology, finance, environmental, and international guidance and best practices. John Wiley \& Sons.

Terra, D. M. (2015). Compliance: percepção dos administradores de mpes. 127 fl. Dissertação (mestrado em Administração das Micro e Pequenas Empresas). Programa de Mestrado em Administração da Faculdade Campo Limpo Paulista, Campo Limpo Paulista, São Paulo, 2015.

The Organization for Economic Co-operation and Development. (2013). Anti-Corruption Ethics and Compliance Handbook for Busines. Recuperado em 16 novembro, 2016, de http://www.oecd.org/corruption/Anti-corruptionethicscompliancehandbook.pdf.

The Organization for Economic Co-operation and Development. (2010). Good Practice Guidance on Internal Controls, Ethics and Compliance. Recuperado em 16 novembro, 2016, de http://www.oecd.org/investment/anti-bribery/anti-briberyconvention/44884389.pdf.

Trapp, H.L.A.F. (2015). Compliance na Lei Anticorrupção: Uma análise da aplicação do art. 7º, VIII, da Lei 12.846/2013. Revista Boletim Jurídico, ed. 1237, Recuperado em 23 abril, 2016, de http://www.boletimjuridico.com.br/m/texto.asp?ld=3969.

United Kingdom. Uk Bribery Act 2010. Lei Anticorrupção Inglesa. Recuperado em 16 abril, 2016, de http://www.legislation.gov.uk/ukpga/2010/23/contents.

United States of America - Department of Justice. A Resource Guide to the U.S Foreign Corrupt Practices Act. Recuperado em 30 abril, 2016, de https://www.justice.gov/sites/default/files/criminal-fraud/legacy/2015/01/16/guide.pdf.

United States of America - United States Sentencing Comission. Federal Sentencing Guidelines. Recuperado em 30 abril, 2016, de http://www.ussc.gov/sites/default/files/pdf/guidelinesmanual/2012/manual-pdf/Chapter_8.pdf.

Wellner, P. A. (2005). Effective compliance programs and corporate criminal prosecutions. Cardozo L. Rev., (27), p. 497-528, Recuperado em 16 novembro, 2016, de http://www.friedfrank.com/sitefiles/Publications/CDB6714353B1B712D3A5DB85F508483E.pdf.

Xavier, C. P. G (2015). Programas de Compliance anticorrupção no contexto da Lei 12.849/2013: Elementos e Estudo de Caso. f. 100. Dissertação (mestrado em Direito). São Paulo: Fundação Getúlio Vargas. 